\title{
Eukaryote-specific rRNA expansion segments function in ribosome biogenesis
}

\author{
MADHUMITHA RAMESH and JOHN L. WOOLFORD JR. \\ Department of Biological Sciences, Carnegie Mellon University, Pittsburgh, Pennsylvania 15232, USA
}

\begin{abstract}
The secondary structure of ribosomal RNA (rRNA) is largely conserved across all kingdoms of life. However, eukaryotes have evolved extra blocks of rRNA sequences, relative to those of prokaryotes, called expansion segments (ES). A thorough characterization of the potential roles of ES remains to be done, possibly because of limitations in the availability of robust systems to study rRNA mutants. We sought to systematically investigate the potential functions, if any, of the ES in 25S rRNA of Saccharomyces cerevisiae by deletion mutagenesis. We deleted 14 of the 16 different eukaryote-specific ES in yeast 25S rRNA individually and assayed their phenotypes. Our results show that all but two of the ES tested are necessary for optimal growth and are required for production of $25 \mathrm{~S}$ rRNA, suggesting that ES play roles in ribosome biogenesis. Further, we classified expansion segments into groups that participate in early nucleolar, middle, and late nucleoplasmic steps of ribosome biogenesis, by assaying their pre-rRNA processing phenotypes. This study is the first of its kind to systematically identify the functions of eukaryote-specific expansion segments by showing that they play roles in specific steps of ribosome biogenesis. The catalog of phenotypes we identified, combined with previous investigations of the roles ribosomal proteins in large subunit biogenesis, leads us to infer that assembling ribosomes are composed of distinct RNA and protein structural neighborhood clusters that participate in specific steps of ribosome biogenesis.
\end{abstract}

Keywords: rRNA; eukaryote; ribosome; protein-RNA; ribosome assembly; expansion segments

\section{INTRODUCTION}

A universal core secondary structure for ribosomal RNA (rRNA) has been identified across all kingdoms of life (Veldman et al. 1981; Clark et al. 1984; Gutell et al. 1993). However, eukaryotic rRNA has additional blocks of sequences relative to prokaryotic rRNA designated as "expansion segments" (ES) (Ware et al. 1983; Clark et al. 1984; Hassouna et al. 1984). These ES are interspersed throughout the various domains of rRNA secondary structure core at specific, conserved sites (Fig. 1A). They exhibit a striking degree of variability in multiple respects: not all ES are found in all species, they vary in their length and sequence both within and among different species; and they even vary between different rRNA repeats of the same organism (Gonzalez et al. 1985). Hence, ES also have been referred to as variable regions and divergent domains (Gerbi 1996). However, the secondary structures of ES exhibit less variability. In eukaryotes, there are a total of $12 \mathrm{ES}$ in the small subunit rRNA $\left(\mathrm{ES}_{\mathrm{S}}\right)$ and 41 in the large subunit rRNA $\left(\mathrm{ES}_{\mathrm{L}}\right)$ compared to prokaryotic Escherichia coli rRNA. However, only a subset of these ES are present in any given eukaryote species. Though ES

Corresponding author: jw17@andrew.cmu.edu

Article published online ahead of print. Article and publication date are at http://www.rnajournal.org/cgi/doi/10.1261/rna.056705.116. have been a continued focus of research, their inherent variability and the limited availability of robust systems to study rRNA mutations have impeded our understanding of their functions.

Recent high-resolution cryo-EM and crystal structures of eukaryotic ribosomes (Spahn et al. 2001; Taylor et al. 2009; Armache et al. 2010; Ben-Shem et al. 2011; Jenner et al. 2012; Anger et al. 2013) have considerably improved our understanding of ES, yielding interesting insights (Fig. 1B). ES are responsible for a majority of the $40 \%$ increase in the size of ribosomes from prokaryotes to eukaryotes (Gerbi 1996; Jenner et al. 2012). Their size and the number of helical branches seem to increase progressively in higher eukaryotes (Fig. 1C; Michot and Bachellerie 1987; Michot et al. 1990). Eukaryotic rRNA ES of the large ribosomal subunit are located predominantly in two clusters on the side of the ribosome exposed to the solvent, although they are distributed across the different domains of rRNA secondary structure (Fig. 1A,B). Importantly, ES do not directly interrupt the functional centers of ribosomes (Fig. 1B; Gerbi 1996; Armache

(C) 2016 Ramesh and Woolford This article is distributed exclusively by the RNA Society for the first 12 months after the full-issue publication date (see http://rnajournal.cshlp.org/site/misc/terms.xhtml). After 12 months, it is available under a Creative Commons License (Attribution-NonCommercial 4.0 International), as described at http://creativecommons.org/licenses/ by-nc/4.0/. 


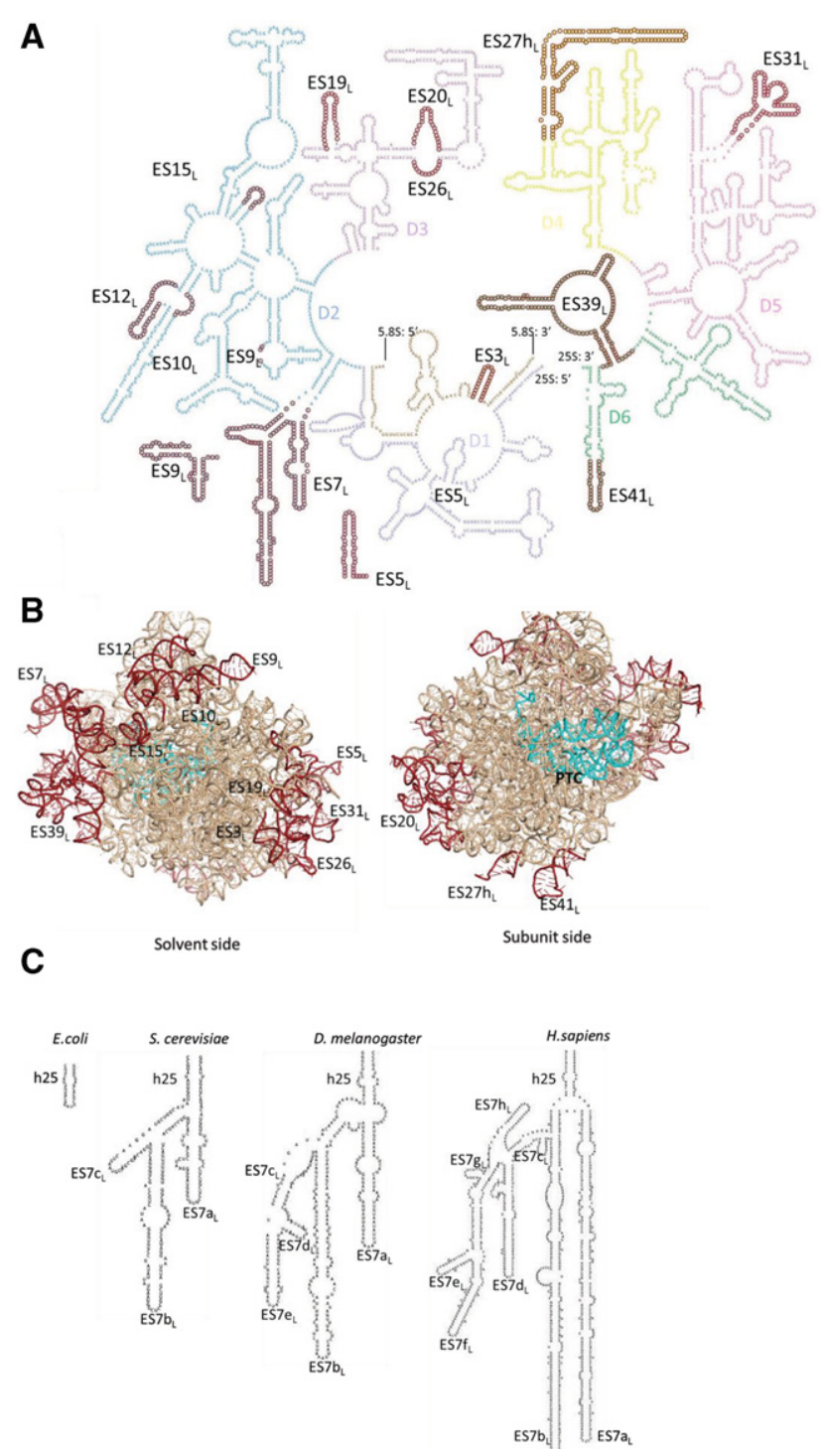

FIGURE 1. Ribosomal RNA expansion segments in the yeast large ribosomal subunit. (A) Secondary structure of Saccharomyces cerevisiae large subunit ribosomal RNA (rRNA). Different domains are marked by different colors. The ES deletions studied here (14 of the 16 ES in yeast LSU rRNA) are highlighted by maroon circles and labeled. (B) Pymol representation of the large subunit rRNA. ES deletions studied are highlighted in maroon as in $A$ and labeled. The peptidyl transferase center (PTC) is shown in cyan. (C) ES progressively increase in size and complexity in eukaryotes. Helix 25 (h25) is shown as an example to demonstrate the increase in sequence length (not to scale) and the number of helical branches of ES7 L from E. coli through Homo sapiens.

et al. 2010; Ben-Shem et al. 2011; Anger et al. 2013). Thus, ribosomes of higher eukaryotes have been described as having a conserved core, with a shell consisting of a flexible outer layer formed by the helical insertions of the ES and eukaryote-specific portions of ribosomal proteins (r-proteins) (Melnikov et al. 2012). In agreement with an earlier study that suggested that ES are on the surface of the ribosome (Han et al. 1994), their clustering on the solvent-exposed surface of the ribosome potentially enables a multitude of mo- lecular interactions by these ES to modulate ribosome assembly and function.

The most interesting question about ES pertains to their function. It has not been determined fully if ES are innocuous, dispensable insertions that arose during the course of evolution, or instead, whether they have specific functions in the cell. Various studies have been carried out to investigate whether ES might have roles in ribosome biogenesis or function. In one example, replacing the ES19 $\mathrm{L}$ region of yeast $25 \mathrm{~S}$ rRNA with E. coli or mouse ES sequences was tolerated without apparent loss of function (Musters et al. 1991). Likewise, insertions in the $\mathrm{ES}_{\mathrm{L}}$ and $\mathrm{ES}_{\mathrm{S}}$ regions of yeast and insertions near the $3^{\prime}$ end of the large subunit rRNA in Tetrahymena were functional (Musters et al. 1989, 1990; Sweeney and Yao 1989). In contrast, a deletion of Tetrahymena ES27 $7_{\mathrm{L}}$ prevented growth (Sweeney et al. 1994). Interestingly, the ES27 $7_{L}$ deletion could be rescued by replacement with ES27 $7_{\mathrm{L}}$ sequences from another organism, but not with random sequences, leading the authors to hypothesize that these regions share an essential function that is not reflected in their primary sequences. Further evidence that ES might carry out specific functions was revealed by deletion mutagenesis of yeast $\mathrm{ES}_{\mathrm{L}}$ and ES27 $\mathrm{L}$. The larger deletion mutations of these ES were lethal due to defects in ribosome biogenesis (Jeeninga et al. 1997). For those ES that are essential, several studies supported the notion that their secondary structure is more important for their function than their sequence (Sweeney et al. 1994; Jeeninga et al. 1997).

The high degree of variability observed in expansion segment sequences and their observed location away from the functional centers of ribosomes suggest that they may not directly participate in universally conserved core aspects of translation. However, they could fine-tune translation, potentially in a species-specific manner (Gao et al. 2005; Hashem et al. 2013). For example, some small subunit ES show complementarity with mRNAs, raising the possibility that ES might affect their translation (Gonzalez et al. 1988). Furthermore, it has been suggested that ES could contribute to the functional heterogeneity of Plasmodium ribosomes (Gunderson et al. 1987; Waters et al. 1995). More recent structural studies have identified other potential roles for ES: (i) ES31 $1_{\mathrm{L}}$ and ES41 $\mathrm{L}$ form additional eukaryote-specific intersubunit bridges in yeast (Spahn et al. 2001); (ii) ES6 ${ }_{S}$, located near the mRNA entry and exit sites, and ES27 $7_{\mathrm{L}}$, located near the polypeptide exit tunnel, have been suggested to facilitate the access of translation factors to ribosomes (Beckmann et al. 2001; Armache et al. 2010; Anger et al. 2013); (iii) ES24 L was proposed to play a role in cotranslational protein localization (Halic et al. 2004). Despite the growing body of evidence indicating the importance of ES in ribosome biogenesis and function, a comprehensive deletion mutagenesis survey that could yield valuable insights into the functions of ES has been lacking.

Here, we systematically investigated the functions of $14 \mathrm{ES}$ of the large subunit rRNA of S. cerevisiae ribosomes, by 
deleting them individually and studying the mutant phenotypes. We found that 12 of the ES studied are necessary for optimal growth, suggesting that the majority of ES play indispensable functions. The steady-state levels of mature $25 \mathrm{~S}$ rRNA are severely diminished in all of the mutants that exhibit a growth deficiency, suggestive of improper biogenesis of $25 \mathrm{~S}$ rRNA. We further categorized ES mutants into early nucleolar, middle, and late nuclear phenotypic groups, based on their pre-rRNA processing phenotype. The observed functional clustering pattern of these rRNA ES functions in the structure of the ribosome resembles those reported for r-proteins (Gamalinda et al. 2014), indicating that distinct neighborhoods in the assembling ribosome might be involved in distinct steps of ribosome biogenesis. We also discuss potential models by which eukaryote-specific protein and RNA elements in the ribosome might have coevolved to perform functions in ribosome biogenesis. Taken together, our data suggest that many ES are indispensable, demonstrate potential functions for ES in ribosome biogenesis, and explain some of their distinct roles in ribosome biogenesis.

\section{RESULTS AND DISCUSSION}

\section{ES mutants and the system to assay their phenotype}

The presence of around 200 copies of rDNA genes in the yeast genome makes analysis of rDNA mutants challenging. In order to assay the in vivo phenotype of ES deletions, we used a strain carrying a temperature sensitive mutation in a subunit of RNA polymerase I (NOY504), the enzyme that exclusively transcribes rDNA (Nogi et al. 1993). We transformed this strain with a plasmid (pWL160) (Liang and Fournier 1997; Cole and LaRiviere 2008) containing an rDNA unit driven by Pol II from a GAL7 promoter. At the permissive temperature, endogenous chromosomally derived WT rRNA is made as well as rRNA from the plasmid. However, at the restrictive temperature, transcription of chromosomal rDNA is shut off, and the only source of rRNA is the plasmid (pWL160) (Supplemental Fig. 1A). This plasmid contains oligonucleotide tags within $\mathrm{ES}_{\mathrm{L}}$ of $25 \mathrm{~S}$ rRNA and $\mathrm{ES}_{\mathrm{S}}$ of $18 \mathrm{~S}$ rRNA, which permit us to differentiate plasmid-derived rRNA from residual endogenous rRNA.

We introduced 14 ES deletion mutations into plasmidborne $25 \mathrm{~S}$ rDNA individually and assayed each for effects on ribosome biogenesis. Although reported nucleotide definitions of ES can vary (Gerbi 1996; Taylor et al. 2009; Armache et al. 2010; Anger et al. 2013), the exact ES deletions examined in this study are indicated in Supplemental Table 1 and highlighted in Figure 1. In cases where an ES deletion involved truncating a helix in the middle, we introduced a tetraloop to maintain stability of the helix and to minimize secondary effects. With the exception of ES4 $4_{\mathrm{L}}$ and ES24 $4_{\mathrm{L}}$ (Peculis and Greer 1998; Cote and Peculis 2001; Halic et al. 2004), we have examined nearly all of the ES of the large sub- unit rRNA in yeast. Also, we studied a partial deletion of $\mathrm{ES}_{2} 7_{\mathrm{L}}$, as marked in Figure 1, denoted henceforth as $\mathrm{ES} 2 \mathrm{~h}_{\mathrm{L}}$ to represent the deletion of the helix.

\section{Most $\mathrm{ES}_{\mathrm{L}}$ are necessary for optimal growth}

In order to address whether ES are innocuous, dispensable eukaryotic insertions in rRNA, or are essential, we first assayed the viability of the $14 \mathrm{ES}_{\mathrm{L}}$ deletion mutants by spotting serial dilutions of cultures onto solid growth media (Fig. 2). At the permissive temperature (left panels), when the chromosome-derived WT rRNA is expressed, all of the mutant strains grew well, as expected. When grown at the restrictive temperature (right panels), 12 of the 14 ES deletion mutants exhibited a growth defect compared to the WT control. The growth phenotype of these $12 \mathrm{ES}_{\mathrm{L}}$ deletion mutants closely resembled the no rDNA control. Hence, we refer to them as "lethal" ES mutants or "essential" ES. The slight growth we observe at the restrictive temperature presumably results from the preexisting pool of ribosomes and incomplete shut-off of Pol I. Thus, all of the $\mathrm{ES}_{\mathrm{L}}$ tested, except ES19 ${ }_{\mathrm{L}}$

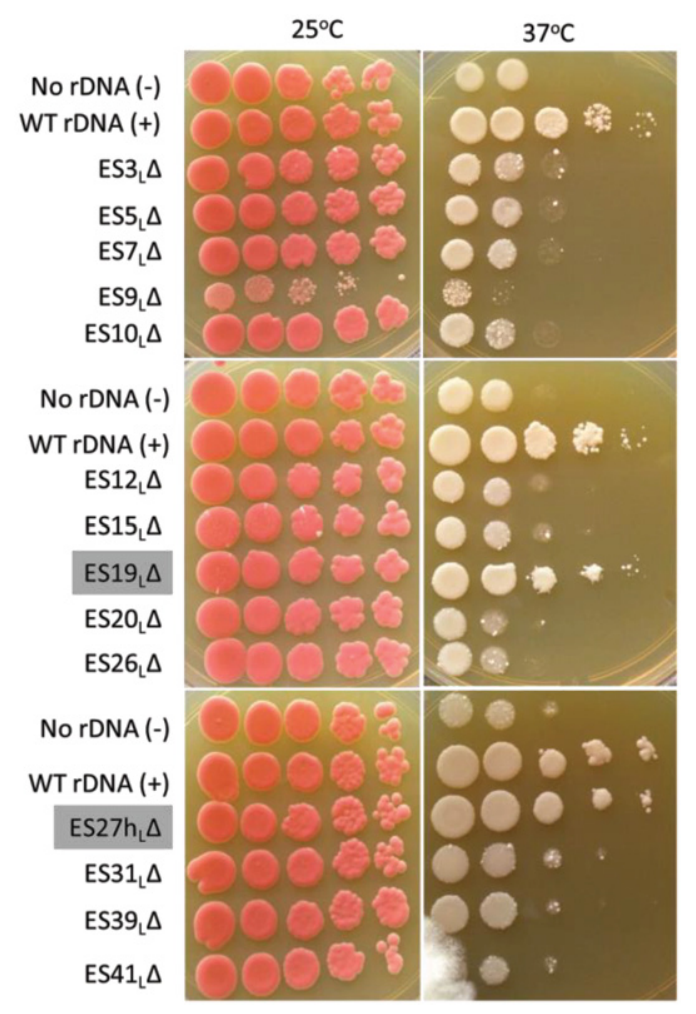

FIGURE 2. Most large subunit ES are necessary for optimal growth. Serial dilutions of log phase yeast cultures were spotted onto solid medium and grown either at the permissive temperature $\left(25^{\circ} \mathrm{C}\right.$, left panels) or at the restrictive temperature $\left(37^{\circ} \mathrm{C}\right.$, right panels). Empty vector ("No rDNA") and wild-type (WT) controls ("WT rDNA") are indicated. The nonessential ES are highlighted with gray labels. Note that a less dense culture of ES9 $9_{\mathrm{L}}$ was used. Similar results were observed across at least three independent biological replicates. 
A

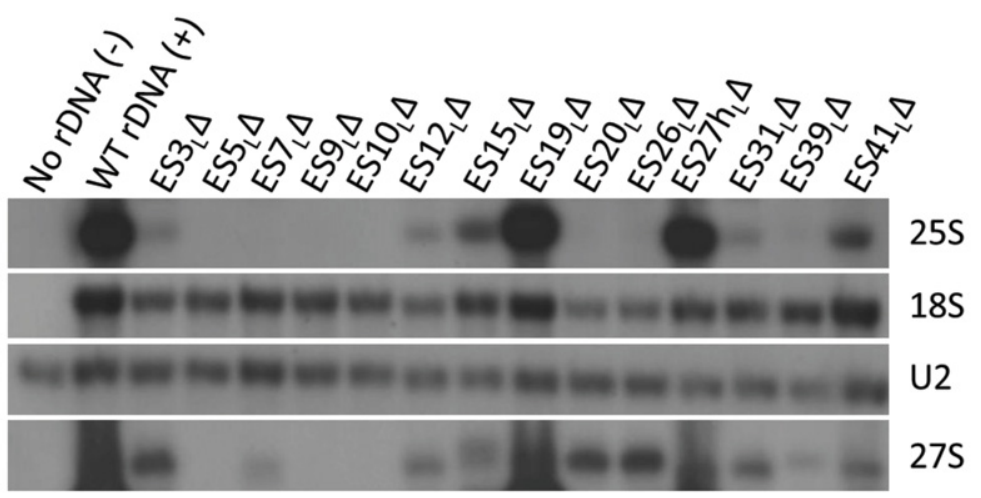

B
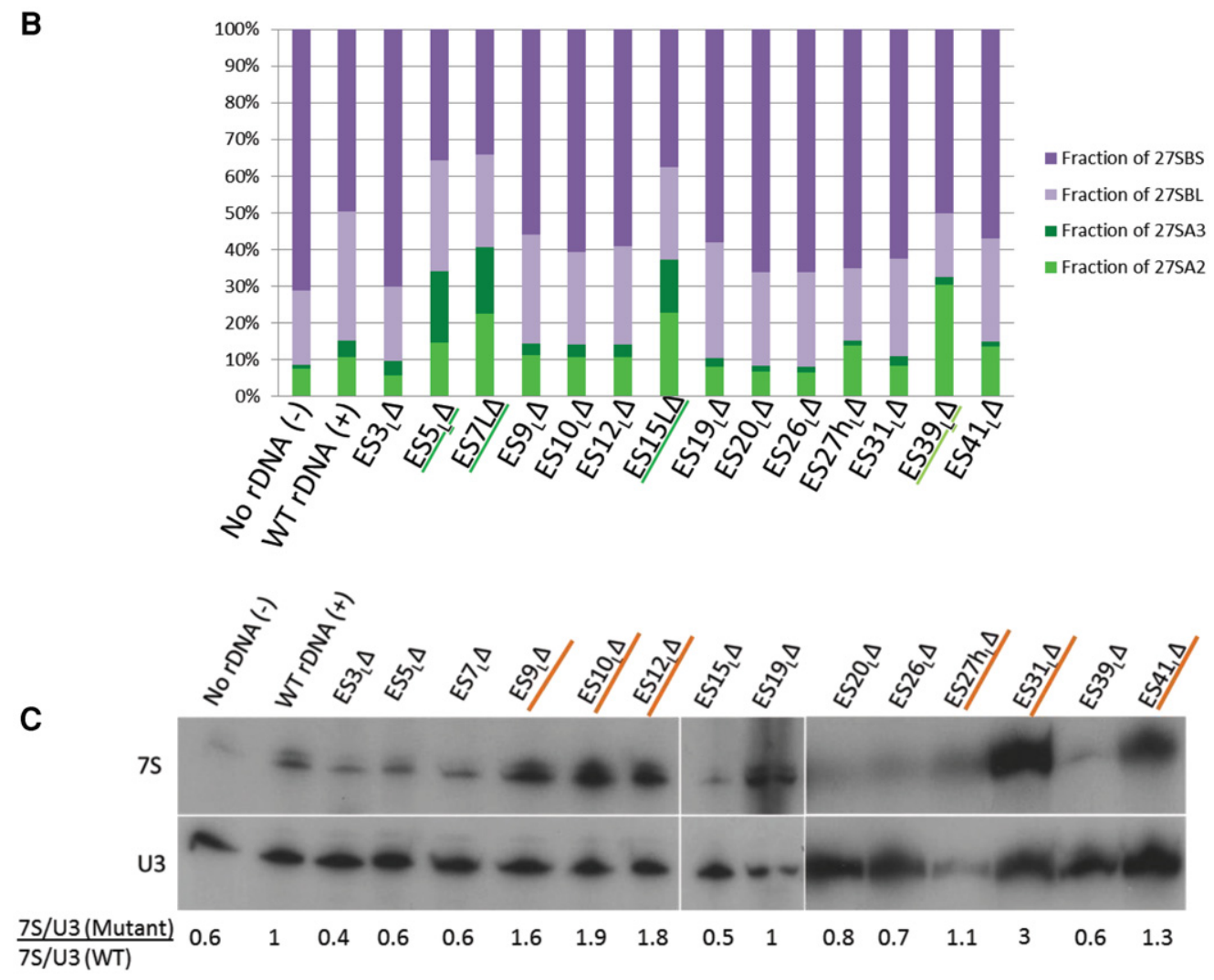

FIGURE 3. (Continued on next page)

and $E S 27 h_{L}$, are necessary for viability, suggesting that most $\mathrm{ES}_{\mathrm{L}}$ play indispensable functions in the cell.

\section{Most $\mathrm{ES}_{\mathrm{L}}$ function in $60 \mathrm{~S}$ subunit biogenesis}

Since ES are eukaryote-specific, we hypothesized that these extra rRNA elements may have evolved to perform eukaryote-specific functions related to the ribosome, including eukaryote-specific features of ribosome biogenesis, nuclear export of nascent ribosomal subunits, and/or eukaryotespecific functions in translation. Since defects in ribosome biogenesis could mask any further defects in translation in these mutants, we decided to begin by assaying for ribosome biogenesis-related functions. To test whether deletions of ES affected synthesis of mature 25S rRNA, we performed a Northern blot of total cellular RNA extracted after shifting to the restrictive temperature (Fig. 3A), and probed for plasmid-derived 25S (first panel, Fig. 3A) and 18S rRNA (second panel, Fig. 3A) using oligonucleotides complementary to the plasmid-specific tag. Strikingly, all the lethal ES deletions resulted in severely diminished, sometimes undetectable levels of mature 25S rRNA, while levels of small subunit $18 \mathrm{~S}$ rRNA remained relatively stable. This confirms that all of the essential ES play a role in the biogenesis of large ribosomal subunits. Those mutants that can produce WT levels of 25S rRNA also exhibit WT-like growth, suggesting that the 
D

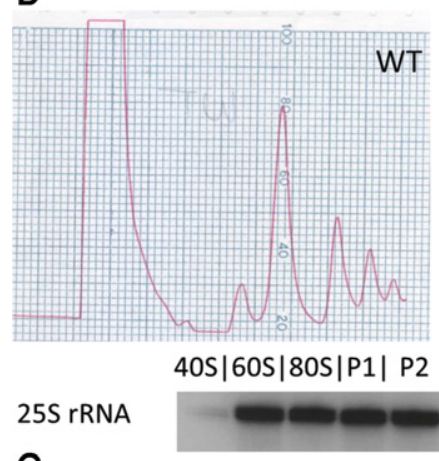

G

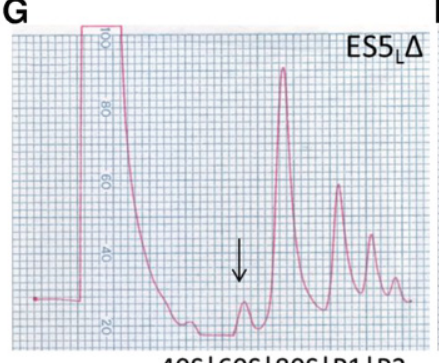

$40 S|60 S| 80 S|P 1| P 2$
E

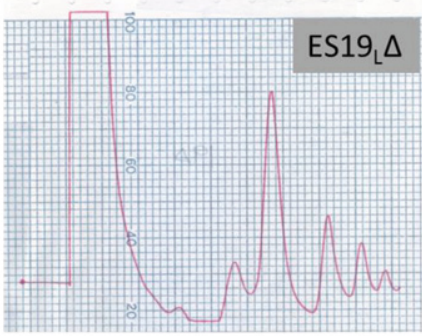

$40 S|60 S| 80 S|P 1| P 2$

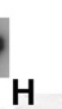

$\mathbf{F}$
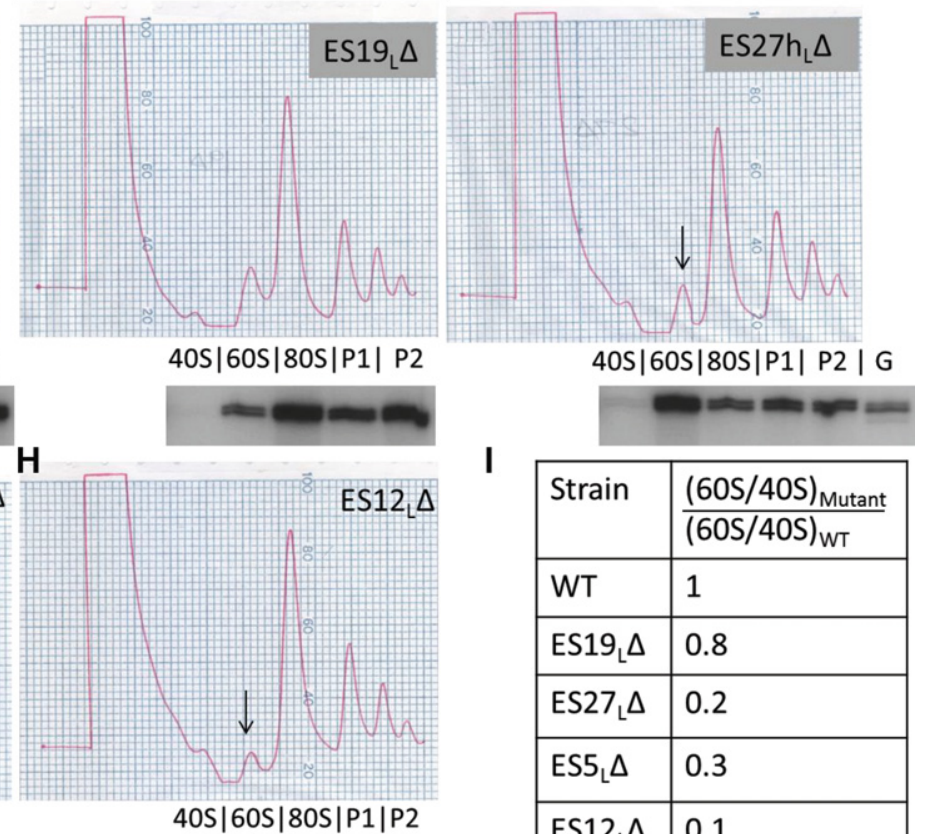

I

\begin{tabular}{|l|l|}
\hline Strain & $\frac{(60 S / 40 S)_{\text {Mutant }}}{(60 S / 40 S)_{W T}}$ \\
\hline WT & 1 \\
\hline ES19 $\Delta$ L & 0.8 \\
\hline$E S 27_{L} \Delta$ & 0.2 \\
\hline$E S 5_{L} \Delta$ & 0.3 \\
\hline ES12 ${ }_{L} \Delta$ & 0.1 \\
\hline
\end{tabular}

FIGURE 3. Different ES participate in different steps of large ribosomal subunit assembly. (A) Representative Northern blot of total cellular RNA extracted from ES mutants, probed with various oligonucleotides to detect rRNAs and pre-rRNAs. Similar effects were observed in two different biological replicates. U2 snRNA is the loading control. Only the plasmid-derived 25S, $18 \mathrm{~S}$ and $27 \mathrm{~S}$ rRNAs are detected, since plasmid-specific oligonucleotide tags were used to probe for these rRNA species. (B) Relative steady-state levels of various $27 \mathrm{~S}$ pre-rRNAs $\left(27 \mathrm{SA}_{2}, 27 \mathrm{SA}_{3}, 27 \mathrm{SB}_{\mathrm{L}}, 27 \mathrm{SB}_{\mathrm{S}}\right)$ in ES mutants, assayed by primer extension. After normalizing each lane with respect to the U2 snRNA loading control, the fraction of each species of pre-rRNA $\left(27 \mathrm{SA}_{2}, 27 \mathrm{SA}_{3}, 27 \mathrm{SB}_{\mathrm{L}}\right.$, or $\left.27 \mathrm{SB}_{\mathrm{S}}\right)$ was calculated as a percentage of total $27 \mathrm{~S}$ pre-rRNA in that lane $\left(27 \mathrm{SA}_{2}+27 \mathrm{SA}_{3}+27 \mathrm{SB}_{\mathrm{L}}+27 \mathrm{SB}_{\mathrm{S}}\right)$. 27SA processing intermediates are shown in shades of green $\left(27 \mathrm{SA}_{2}\right.$ is light green and $27 \mathrm{SA}_{3}$ is dark green). $27 \mathrm{SB}$ processing intermediates are shown in shades of purple $\left(27 \mathrm{SB}_{\mathrm{L}}\right.$ is light purple and $27 \mathrm{SB}_{\mathrm{S}}$ is dark purple). An increase in green $27 \mathrm{SA}$ pre-rRNA and a concomitant decrease in purple $27 \mathrm{SB}$ pre-rRNA are diagnostic of an "early" ES mutant. Note that the levels of pre-rRNA species observed here are a combination of both residual endogenous WT and plasmid-derived mutant pre-rRNA. Shown here are the mean values from three biological replicates. For more details and error bars, see Supplemental Figure 3. (C) Northern blot of total cellular RNA extracted from ES mutants, probed with oligonucleotides that hybridize to 7S prerRNA. U3 snoRNA is the loading control. Shown below the blot is the fold increase of 7S pre-rRNA in mutants compared to WT, normalized for loading. A mutant that has a ratio of $>1$ accumulates $7 \mathrm{~S}$ pre-rRNA (characteristic of late-acting ES, highlighted in orange), and a mutant that has a ratio of $<1$ has decreased levels of $7 \mathrm{~S}$ pre-rRNA (seen in early or middle phenotypes). $(D-I)$ Sucrose gradient centrifugation to assay defects in 60 S ribosomal subunit biogenesis in WT cells $(D)$, viable ES mutants $(E, F)$, and representative lethal ES mutants $(G, H)$. Arrows shown in $G, H$, and $F$ indicate the decreased 60S subunit peak in the lethal ES mutants and in the viable $\mathrm{ES}_{2} 7 \mathrm{~h}_{\mathrm{L}} \Delta$ mutant, respectively, compared to WT $(B)$. In this assay, defects in $60 \mathrm{~S}$ subunit biogenesis are discerned exclusively by a decreased $60 \mathrm{~S}$ peak and a decreased ratio of $60 \mathrm{~S}$ to $40 \mathrm{~S}$ subunits, compared to WT. Plasmid-derived 25S rRNA detected by reverse transcription using an oligonucleotide complementary to the plasmid-specific tag is shown underneath the polysome curves to indicate those fractions that contain plasmid-derived mature $25 \mathrm{~S}$ rRNA. As shown in $F$, a sequencing lane with $\mathrm{G}$ was used to map the $5^{\prime}$ end of $25 \mathrm{~S}$ rRNA. The primer extension assay could not be performed on the $\mathrm{ES}_{\mathrm{L}} \Delta$ mutant in $G$, since this deletion encompasses the plasmid-specific tag, thereby eliminating the binding site of the reverse transcription primer. $(G)$ Ratios of area under the curve of $60 \mathrm{~S} / 40 \mathrm{~S}$ subunits in mutant compared to WT, quantified from $D-H$. All the ES mutants shown, except ES19 $\mathrm{L}_{\mathrm{L}}$, have a multifold decrease in $60 \mathrm{~S} / 40 \mathrm{~S}$ ratio compared to WT, indicative of defects in $60 \mathrm{~S}$ subunit biogenesis.

observed growth deficiencies in the lethal ES mutants primarily result from defective synthesis of stable $25 \mathrm{~S}$ rRNA. In principle, another possibility that could explain this result is that: mature $25 \mathrm{~S}$ rRNA is being made and is subsequently degraded from the translational pool, perhaps by nonfunctional RNA decay (Cole and LaRiviere 2008). However, as shown below, we identified distinct pre-rRNA processing defects in each of our lethal mutants. This suggests that improper biogenesis, perhaps accompanied by turnover during biogenesis as seen before in other ribosomal protein and assembly factor mutants (Sahasranaman et al. 2011; Jakovljevic et al. 2012; Gamalinda et al. 2014), leads to the production of severely decreased levels of stable $25 \mathrm{~S}$ rRNA in the lethal ES mutants.

\section{Various $\mathrm{ES}_{\mathrm{L}}$ function in different steps of ribosome assembly}

Ribosome biogenesis proceeds in a series of pre-rRNA processing steps (Supplemental Fig. 2), which have been used to demarcate the progress of ribosome assembly in biogenesis mutants. Thus, we examined which pre-rRNA processing 
steps are affected in each of the ES mutants by Northern blotting and primer extension. We defined "early"-acting ES as those, when deleted, result in an increase in levels of $27 \mathrm{SA}_{2}$ and $27 \mathrm{SA}_{3}$ pre-rRNA accompanied by a relative decrease in 27SB pre-rRNA. These early processing steps occur in the nucleolus. We defined "late"-acting ES as those, when deleted, result in an increase of $7 \mathrm{~S}$ pre-rRNA, the processing of which begins in the nucleoplasm. ES mutants that result in an increase of $27 \mathrm{~S}$ pre-rRNA that cannot be explained by an increase in 27SA pre-rRNA (while not resulting in an increase in $7 \mathrm{~S}$ pre-rRNA) were categorized as "middle"-acting, presumably resulting in inefficient processing of $27 \mathrm{SB}$ pre-rRNA.

Compared to the WT rDNA control, we observed that the ES39 $\Delta_{\mathrm{L}}$ mutant showed increased levels of $27 \mathrm{SA} \mathrm{A}_{2}$ pre-rRNA relative to later $27 \mathrm{~S}$ pre-rRNA processing intermediates. In addition, $E S 5 \Delta_{\mathrm{L}}, \mathrm{ES} 7 \Delta_{\mathrm{L}}$, and $\mathrm{ES} 15 \Delta_{\mathrm{L}}$ showed a relative increase of the $27 \mathrm{SA}_{3}$ pre-rRNA species accompanied by a concomitant decrease in $27 \mathrm{SB}_{S}$ pre-rRNA. Hence, we categorize $\mathrm{ES} 39 \Delta_{\mathrm{L}}, \mathrm{ES} 5 \Delta_{\mathrm{L}}, \mathrm{ES} 7 \Delta_{\mathrm{L}}$, and ES15 $\Delta_{\mathrm{L}}$ (indicated by text underlined in green in Fig. 3B) as early-acting ES, with $\mathrm{ES} 39 \Delta_{\mathrm{L}}$ affecting $27 \mathrm{SA}_{2}$ processing and the remainder affecting $27 \mathrm{SA}_{3}$ pre-rRNA processing.

Since the 27SB pre-rRNA intermediate is relatively longlived (Kos and Tollervey 2010), and the primer extension assay described above measures the combined levels of endogenous WT rRNA and rRNA derived from the plasmid, we were not able to unequivocally determine increases in 27SB pre-rRNA levels, characteristic of the "middle" steps. However, for several mutants, most noticeably ES3 $\Delta_{\mathrm{L}}$, ES20 $\Delta_{\mathrm{L}}$, and ES26 $\Delta_{\mathrm{L}}$, we were able to infer plasmid-derived 27S $(\mathrm{A}+\mathrm{B})$ pre-rRNA accumulation (Fig. 3A, fourth panel) using the plasmid-specific oligonucleotide complementary to $25 \mathrm{~S}$ rRNA. There are two considerations worth noting here. First, the relative lifetimes of various pre-rRNA intermediates are different (Kos and Tollervey 2010); hence the effects on the long-lived 27SB pre-rRNA may be overrepresented in the steady-state Northern blot assay shown in Figure 3A. Second, it has been shown that preribosomes become increasingly stable as assembly proceeds (Gamalinda et al. 2014). Since this steady-state measurement is indicative of a combination of accumulation and turnover, there may be a bias toward later, more stable particles containing 27SB pre-rRNA. Based on these considerations, we conclude that $\mathrm{ES}_{\mathrm{L}}, \mathrm{ES} 20_{\mathrm{L}}$, and $\mathrm{ES} 26_{\mathrm{L}}$ fall into the "middle"-acting category, which includes those that help in processing of $27 \mathrm{SB}$ pre-rRNA.

To identify "late"-acting ES mutants, we assayed for effects on 7S pre-rRNA processing by Northern blotting of RNAs, using U3 snoRNA as a loading control (Fig. 3C). After normalizing for loading, we calculated the ratio of levels of $7 \mathrm{~S}$ pre-rRNA in the mutants to $7 \mathrm{~S}$ pre-rRNA in the WT control in the same blot with the same exposure. Using this assay, we identified $\mathrm{ES} 9_{\mathrm{L}}, \mathrm{ES} 10_{\mathrm{L}}, \mathrm{ES} 12_{\mathrm{L}}, \mathrm{ES} 31_{\mathrm{L}}$, and $\mathrm{ES} 41_{\mathrm{L}}$ among the essential ES with a late-acting function, evident by $7 \mathrm{~S}$ pre-rRNA accumulation when they are deleted. Of these,
ES31 $\Delta_{\mathrm{L}}$ seemed to possess the highest accumulation of $7 \mathrm{~S}$ pre-rRNA. Those ES mutants that result in an accumulation of $7 \mathrm{~S}$ pre-rRNA are underlined in orange in Figure 3C. In addition, we observed that $7 \mathrm{~S}$ pre-rRNA levels decreased in the early- and middle-acting ES, consistent with inefficient processing of upstream $27 \mathrm{~S}$ pre-rRNAs.

\section{Viable ES mutants can synthesize mature 25S rRNA}

To further understand the effect of nonlethal ES mutations, we performed sucrose gradient fractionation to separate free ribosomal subunits and polyribosomes in the viable ES mutants (Fig. 3E,F) as well as in representative lethal ES mutants (Fig. 3G,H). These mutant strains, along with a WT control (Fig. 3D), were grown at the permissive temperature and then shifted to the restrictive temperature for $6 \mathrm{~h}$ prior to preparing extracts for gradient analysis. If any of these mutants had defects in $60 \mathrm{~S}$ subunit biogenesis, we expect that levels of free $60 \mathrm{~S}$ subunits will be decreased compared to $40 \mathrm{~S}$ subunits (reflected as a decrease in $60 \mathrm{~S} / 40 \mathrm{~S}$ subunit ratio, in comparison with the WT strain, Fig. 3I). Among the viable ES mutants, ES19 $\Delta_{\mathrm{L}}$ resembled the WT control. However, the nonlethal ES27h $\Delta_{\mathrm{L}}$ exhibited a decreased 60S peak and a decrease in the $60 \mathrm{~S} / 40 \mathrm{~S}$ ratio, indicating that this part of ES27 $7_{\mathrm{L}}$ may possibly play a role that is dispensable in $60 \mathrm{~S} \mathrm{sub-}$ unit biogenesis. This could potentially reflect a slight inefficiency in the 7S pre-rRNA processing step observed in this mutant (Fig. 3C). Processing of 7S pre-rRNA has been shown to be dispensable for the normal function of the ribosome (Rodriguez-Galan et al. 2015), hence it is not surprising that this mutant yields a slight $7 \mathrm{~S}$ pre-rRNA processing defect while it exhibits no growth defect. Consistent with their inability to make mature $25 \mathrm{~S}$ rRNA, the lethal mutants ES5 $\Delta_{\mathrm{L}}$ and ES12 $\Delta_{\mathrm{L}}$ showed a decreased $60 \mathrm{~S}$ peak and exhibited a multifold decrease in the $60 \mathrm{~S} / 40 \mathrm{~S}$ ratio, compared to the WT control. We surmise that other lethal $\mathrm{ES}_{\mathrm{L}}$ deletion mutants would exhibit a similar effect in this assay. Note that, under the conditions of this assay, synthesis of new WT rRNA from the chromosome is largely blocked. However, a preexisting pool of WT rRNA remains in these cells. Thus, we do not expect to observe other characteristic indicators of a $60 \mathrm{~S}$ subunit biogenesis defect such as a diminished $80 \mathrm{~S}$ peak or halfmer polysomes.

To further confirm that the observed effects arose from the plasmid-derived rRNA, we performed a primer extension assay using the plasmid-specific $25 \mathrm{~S}$ rRNA tag. We detected the $5^{\prime}$ end of $25 \mathrm{~S}$ rRNA in the 60S, 80S, and polyribosome fractions $\mathrm{P} 1$ and $\mathrm{P} 2$ of the WT, ES19 $\Delta_{\mathrm{L}}$ and $\mathrm{ES} 27 \mathrm{~h} \Delta_{\mathrm{L}}$ extracts (but not in the free $40 \mathrm{~S}$ subunit fraction, as expected). This result is consistent with our observation that the viable mutants can synthesize plasmid-derived mature $25 \mathrm{~S}$ rRNA at near WT levels. Conversely, in the lethal mutant ES12 $\Delta_{\mathrm{L}}$, we were not able to detect plasmid-derived 25S rRNA in any fraction, indicating severe defects in large subunit biogenesis. 
The location of ES in specific neighborhoods of the 605 subunit correlates with their ribosome assembly phenotype

Putting the results together, we have discussed additional information about each $\mathrm{ES}_{\mathrm{L}}$ individually in Supplemental Data. We have highlighted relevant previous information about that particular $\mathrm{ES}_{\mathrm{L}}$ and interpreted the results of our $\mathrm{ES}_{\mathrm{L}}$ mutations in light of the contacts they make with r-proteins in mature subunits. Having developed a catalog of ES phenotypes, we sought to understand whether the reported phenomenon of clustering of r-proteins according to their function in successive steps of ribosome biogenesis (Gamalinda et al. 2014) extends to ES. To visualize their locations, we color-coded early, middle, and lateacting ES and r-proteins in shades of green, purple, or orange, respectively (Fig. 4). Recognizing that ES are localized only to the periphery of the solvent interface of the large subunit, as opposed to $r$-proteins that are distributed more evenly, we observed that the early-acting ES localize to the equatorial belt, the middle-acting ES localize near the bottom third of the subunit, and the lateacting ES localize near the central protuberance (Fig. 4A). This pattern of ES clustering according to their function in successive steps of ribosome biogenesis is reminiscent of the functional clustering of $\mathrm{r}$-proteins reported previously (Fig. 4B,C).

In order to explore this further, we have represented each of the ES studied, along with the r-proteins that make contact with that particular ES, in mature yeast ribosomes (Supplemental Fig. 4A-C; Supplemental Table 1; BenShem et al. 2011). It is apparent that in the majority of cases, the phenotype caused by deletion of a particular ES corresponds to the phenotype caused by depletion of the r-proteins that it contacts.

This functional clustering of both rRNA and r-proteins on the solvent interface has interesting implications. First, it adds credence to the possibility that as the ribosome is constructed, there may be neighborhood-specific effects on pre-rRNA processing, folding, and stability. A hierarchy of r-protein assembly exists where r-proteins that participate in specific steps cluster to specific neighborhoods. Our results suggest that this hierarchy might extend to RNA-protein neighbor-

\section{EARLY | MIDDLE | LATE} pre-rRNA phenotypes

B

C
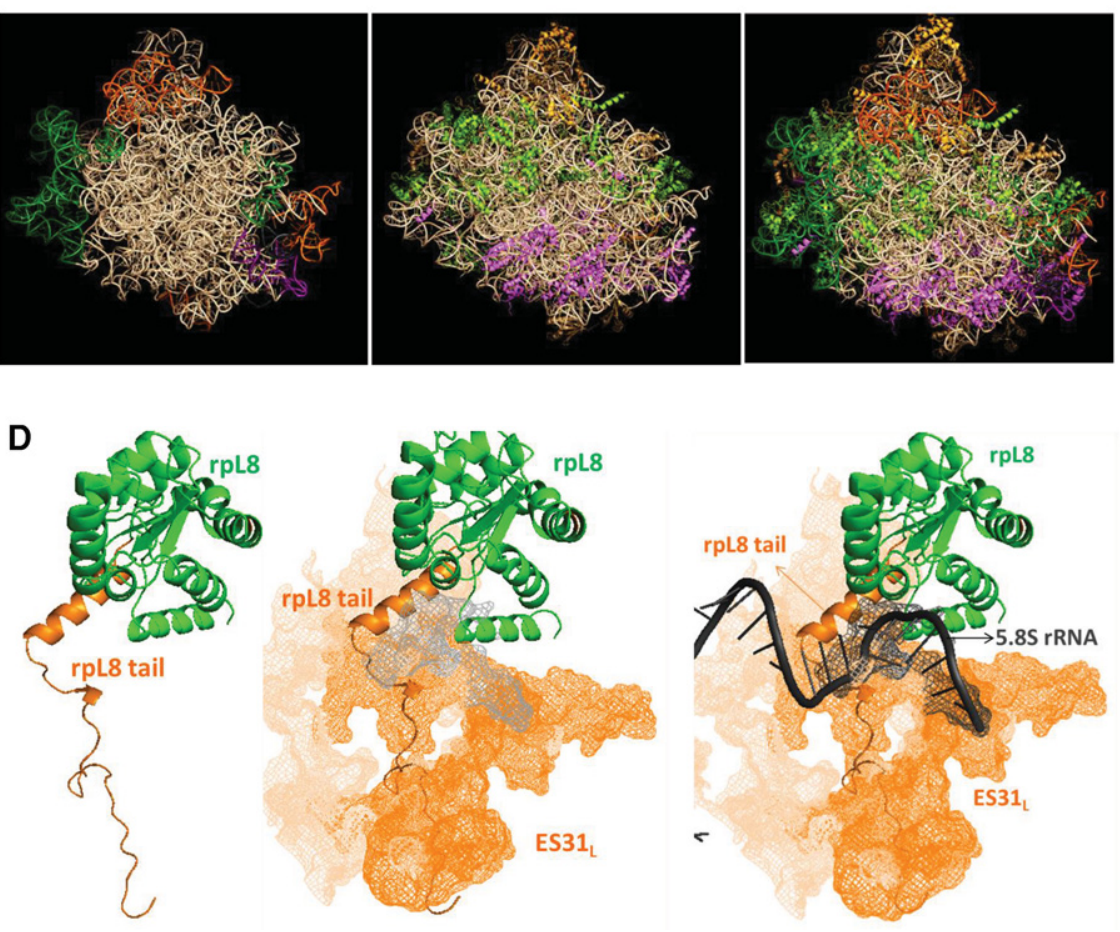

FIGURE 4. A model for ES functions and neighborhood-specific effects in ribosome biogenesis. (A) Structural model of the large ribosomal subunit showing ES color-coded according to the pre-rRNA processing steps in which they function. Green-colored ES are necessary for 27SA processing (early steps) and localize to the "equatorial belt." Purple-colored ES participate in $27 \mathrm{~S}$ processing, but not specifically 27SA processing (middle steps). These ES localize near the bottom third of the solvent side of the ribosome, near the polypeptide exit tunnel. Orange-colored ES participate in $7 \mathrm{~S}$ pre-rRNA processing (late steps) and localize near the central protuberance. (B) The same color scheme (early, green; middle, purple; late, orange) is used to represent previously known r-protein depletion phenotypes. (C) Superimposition of $A$ on $B$. See Results and for details of the model. (D) A case study of eukaryote-specific r-protein-rRNA elements. The left panel shows the body of r-protein L8 (in green, consistent with its "early" role assembly) and its eukaryote-specific N-terminal extension (in orange, consisten with its "late" role in ribosome assembly). The center panel shows the contact established between panel shows the $3^{\prime}$ end of $5.8 \mathrm{~S}$ rRNA (dark gray) to denote the terminal site of pre-rRNA processing of ITS2 by the exosome. ES31 $\mathrm{L}$ is in close contact with the $3^{\prime}$ end of $5.8 \mathrm{~S}$ rRNA.

hoods, not simply r-proteins, in the assembling ribosome (Shajani et al. 2011; Woodson 2011; Gamalinda et al. 2014).

\section{A case study of ES31 $\mathrm{L}$}

Perhaps the only glaring exception to the neighborhood-specific separation of ribosome assembly functions of ES arises from the phenotype of the deletion of ES31 $1_{\mathrm{L}}$. Although the location of ES31 $\mathrm{L}$ in the "equatorial belt" predicts that it might have an early-acting phenotype, in fact, we observed a predominantly late phenotype for $\mathrm{ES} 31 \Delta_{\mathrm{L}}$. To better understand this apparent discrepancy, we looked at this area of the ribosome in more detail to build a model for the function of ES31 $\mathrm{L}$ based on its contact with r-protein L8 (Fig. 4D). 
Depletion of L8 causes an early phenotype (Jakovljevic et al. 2012), consistent with its location in the equatorial belt. On the other hand, deletion of the eukaryote-specific N-terminal extension of L8 that intimately contacts $\mathrm{ES} 31_{\mathrm{L}}$, causes a later, $7 S$ pre-rRNA accumulation phenotype (Tutuncuoglu et al. 2016), which is similar to the phenotype that $E S 31 \Delta_{\mathrm{L}}$ exhibits. Thus, what initially appeared to be an exception to the neighborhood-specific phenotype rule, turned out to be an informative sign about how eukaryote-specific RNAprotein elements that contact each other, exhibit similar functions in ribosome assembly. Strikingly, the $3^{\prime}$ end of $5.8 \mathrm{~S}$ rRNA, where processing of $7 \mathrm{~S}$ pre-rRNA terminates during late steps of $60 \mathrm{~S}$ subunit biogenesis, is encompassed by $\mathrm{ES} 31_{\mathrm{L}}$. This indicates that potential proximity effects may be at play, resulting in a late processing phenotype in this mutant.

\section{A potential model for ES function: ES may have coevolved as scaffolds for eukaryote-specific protein elements in the assembling ribosome}

In this study, we observed that there is functional similarity in ribosome biogenesis between ES rRNA and neighboring rproteins. Furthermore, it has been previously pointed out that eukaryotic RNA elements and eukaryotic r-proteins and their extensions localize together on the solvent-exposed side of the ribosome and make numerous contacts with each other (Ben-Shem et al. 2011). Several eukaryote-specific assembly factors such as Rlp7, Arx1, Rrp5, and Nop7 bind to ES (Granneman et al. 2011; Bradatsch et al. 2012; Babiano et al. 2013; Dembowski et al. 2013; Lebaron et al. 2013). Combined with our observation about similar functions in ribosome assembly for eukaryote-specific ES31 $\Delta_{\mathrm{L}}$ and the eukaryote-specific extension of L8, these lines of evidence raise the possibility that eukaryote-specific protein-RNA elements may have coevolved, and perform similar functions in the cell. It is tempting to suggest that ES may have coevolved with the r-proteins in order to provide a scaffold for binding of many eukaryote-specific protein elements, including assembly factors, r-proteins and their extensions, and translation factors. Once extant technical challenges are overcome, mutations in eukaryote-specific regions of proteins in tandem with eukaryote-specific RNA elements may lead to interesting insights about the function of these eukaryotic elements.

In addition, using this study as a starting point and adopting an evolutionary standpoint, it would be interesting to replace yeast ES with those of other eukaryotes and assay for rescue of those phenotypes with corresponding eukaryotic proteins, to understand how these ES have continued to evolve. Also, whether the precise functions of specific ES in biogenesis and translation are conserved in other eukaryotes remains to be investigated. Further, having established that these ES participate in ribosome biogenesis, it is tempting to ask if rRNA ES might play more direct eukaryote-specific roles in pre-rRNA processing or folding, in addition to serving as eukaryote-specific scaffolds.

In summary, we have shown that most eukaryote-specific rRNA ES of the large ribosomal subunit are indispensable and participate in various steps of ribosome biogenesis. While complementing a vast body of literature about the functions of r-proteins, this study settles the long-standing debate about the dispensability of ES and identifies a catalog of specific functions of these eukaryote-specific insertions in ribosome biogenesis. These results provide a framework for further investigation of eukaryote-specific features of ribosome assembly and function and pave the way for many further studies of the evolution, structure, biogenesis and function of the ribosome.

\section{MATERIALS AND METHODS}

\section{Construction and growth of yeast strains}

As the parent strain, we used NOY504 (JWY10423, MATa rrn4:: LEU2 ade2-101 ura3-1 trp1-1 leu2-3,112 his3-11 canl-100), which lacks the Rpa12 subunit of RNA polymerase 1, rendering it temperature sensitive for growth (a gift from the Nomura laboratory) (Nogi et al. 1993). This strain was transformed with the plasmid pWL160 (a gift from the M. Fournier laboratory) encoding WT RNA as the positive control, or the pRS314 vector as the "no rDNA" negative control.

Handling of the NOY504-based strains was done essentially as described previously (Henry et al. 1994). Briefly, small cultures of the controls and the mutant yeast strains were grown to log phase at $25^{\circ}$ $\mathrm{C}$ in galactose minimal medium. Cultures were then diluted, and endogenous ribosomal RNA was depleted by shifting cultures to $37^{\circ} \mathrm{C}$ for $6 \mathrm{~h}$. The mid log-phase cells were then harvested by centrifugation at $5000 \mathrm{rpm}$ for $5 \mathrm{~min}$ at $4^{\circ} \mathrm{C}$ for further experiments.

\section{Mutagenesis}

The size and location of ES deletions were devised from definitions of ES insertion regions from Gerbi (1996) and secondary structures derived from comparative analysis (Gutell et al. 1993). Mutagenesis of the ES regions (Supplemental Table 1) was carried out in the pWL160 plasmid using the Quikchange Lightning kit from Agilent Technologies. Mutagenesis was verified by DNA sequencing. Sequences for oligonucleotides used in this study are available upon request.

\section{Growth assays of ES mutants}

Small 5-mL cultures of the control and mutant strains were grown in C-Trp+Gal media overnight. Tenfold serial dilutions of yeast strains bearing either the controls (the positive control is JWY10430: NOY504 with pWL160, and the negative control is JWY10429: NOY504 with pRS314) or one of the 14 mutant plasmids from Supplemental Table 1 (JWY10431-JWY10444) were spotted onto YEPD solid medium starting with an OD of 1 and grown either at $25^{\circ} \mathrm{C}$ and photographed after $3 \mathrm{~d}$, or grown at $37^{\circ} \mathrm{C}$ and photographed after $5 \mathrm{~d}$. 


\section{Sucrose gradient analysis}

Gradient fractionation of preribosomes, ribosomes, and polyribosomes, and subsequent analysis, were carried out as described (Talkish et al. 2014). The image was digitized using the PlotDigitizer software. The area under the curve for each peak was calculated using the trapezoidal rule, and subsequently, 60S/40S ratios were determined using Excel.

\section{RNA analysis}

RNA extraction and subsequent Northern blotting assays were performed as described previously (Horsey et al. 2004). Assaying steady state pre-rRNA processing by primer extension was carried out using the Transcriptor reverse transcriptase (Roche) as described (Talkish et al. 2014).

\section{Illustrations}

The PDB file of the crystal structure of yeast ribosomes (PDB ID: 4V88) (Ben-Shem et al. 2011) was manipulated using PyMol software to produce the structural images (The PyMOL molecular graphics system, version 1.8, Schrodinger LLC). RiboVision software (Bernier et al. 2014) was used for generation of some of the secondary structures shown here.

\section{SUPPLEMENTAL MATERIAL}

Supplemental material is available for this article.

\section{ACKNOWLEDGMENTS}

This work was supported by grants from the National Institutes of Health to J.L.W. (GM028301) and the David Scaife Family Charitable Foundation (141RA01). We thank the members of the Woolford laboratory for critical reading of the manuscript.

Received March 30, 2016; accepted May 9, 2016.

\section{REFERENCES}

Anger AM, Armache JP, Berninghausen O, Habeck M, Subklewe M, Wilson DN, Beckmann R. 2013. Structures of the human and Drosophila 80S ribosome. Nature 497: 80-85.

Armache JP, Jarasch A, Anger AM, Villa E, Becker T, Bhushan S, Jossinet F, Habeck M, Dindar G, Franckenberg S, et al. 2010. Cryo-EM structure and rRNA model of a translating eukaryotic 80S ribosome at 5.5-A resolution. Proc Natl Acad Sci 107: 1974819753.

Babiano R, Badis G, Saveanu C, Namane A, Doyen A, Diaz-Quintana A, Jacquier A, Fromont-Racine M, de la Cruz J. 2013. Yeast ribosomal protein $\mathrm{L} 7$ and its homologue Rlp7 are simultaneously present at distinct sites on pre-60S ribosomal particles. Nucleic Acids Res 41: 9461-9470.

Beckmann R, Spahn CM, Eswar N, Helmers J, Penczek PA, Sali A, Frank J, Blobel G. 2001. Architecture of the protein-conducting channel associated with the translating $80 \mathrm{~S}$ ribosome. Cell 107: 361-372.

Ben-Shem A, Garreau de Loubresse N, Melnikov S, Jenner L, Yusupova G, Yusupov M. 2011. The structure of the eukaryotic ribosome at 3.0 A resolution. Science 334: 1524-1529.
Bernier CR, Petrov AS, Waterbury CC, Jett J, Li F, Freil LE, Xiong X, Wang L, Migliozzi BL, Hershkovits E, et al. 2014. RiboVision suite for visualization and analysis of ribosomes. Faraday Discuss 169: 195-207.

Bradatsch B, Leidig C, Granneman S, Gnadig M, Tollervey D, Bottcher B, Beckmann R, Hurt E. 2012. Structure of the pre-60S ribosomal subunit with nuclear export factor Arxl bound at the exit tunnel. Nat Struct Mol Biol 19: 1234-1241.

Clark CG, Tague BW, Ware VC, Gerbi SA. 1984. Xenopus laevis $28 \mathrm{~S}$ ribosomal RNA: a secondary structure model and its evolutionary and functional implications. Nucleic Acids Res 12: 6197-6220.

Cole SE, LaRiviere FJ. 2008. Chapter 12. Analysis of nonfunctional ribosomal RNA decay in Saccharomyces cerevisiae. Methods Enzymol 449: 239-259.

Cote CA, Peculis BA. 2001. Role of the ITS2-proximal stem and evidence for indirect recognition of processing sites in pre-rRNA processing in yeast. Nucleic Acids Res 29: 2106-2116.

Dembowski JA, Ramesh M, McManus CJ, Woolford JL Jr. 2013. Identification of the binding site of Rlp7 on assembling 60S ribosomal subunits in Saccharomyces cerevisiae. RNA 19: 1639-1647.

Gamalinda M, Ohmayer U, Jakovljevic J, Kumcuoglu B, Woolford J, Mbom B, Lin L, Woolford JL Jr. 2014. A hierarchical model for assembly of eukaryotic $60 \mathrm{~S}$ ribosomal subunit domains. Genes Dev 28: 198-210.

Gao H, Ayub MJ, Levin MJ, Frank J. 2005. The structure of the 80 S ribosome from Trypanosoma cruzi reveals unique rRNA components. Proc Natl Acad Sci 102: 10206-10211.

Gerbi S. 1996. Expansion segments: regions of variable size that interrupt the universal core secondary structure of ribosomal RNA. In Ribosomal RNA structure, evolution, processing, and function in protein biosynthesis (ed. Zimmermann RA, Dahlberg AE), pp. 71-87. Telford-CRC Press, Boca Raton, FL.

Gonzalez IL, Gorski JL, Campen TJ, Dorney DJ, Erickson JM, Sylvester JE, Schmickel RD. 1985. Variation among human 28S ribosomal RNA genes. Proc Natl Acad Sci 82: 7666-7670.

Gonzalez IL, Sylvester JE, Schmickel RD. 1988. Human 28S ribosomal RNA sequence heterogeneity. Nucleic Acids Res 16: 10213-10224.

Granneman S, Petfalski E, Tollervey D. 2011. A cluster of ribosome synthesis factors regulate pre-rRNA folding and $5.8 \mathrm{~S}$ rRNA maturation by the Rat1 exonuclease. EMBO J 30: 4006-4019.

Gunderson JH, Sogin ML, Wollett G, Hollingdale M, de la Cruz VF, Waters AP, McCutchan TF. 1987. Structurally distinct, stage-specific ribosomes occur in Plasmodium. Science 238: 933-937.

Gutell RR, Gray MW, Schnare MN. 1993. A compilation of large subunit (23S and 23S-like) ribosomal RNA structures: 1993. Nucleic Acids Res 21: 3055-3074.

Halic M, Becker T, Pool MR, Spahn CM, Grassucci RA, Frank J, Beckmann R. 2004. Structure of the signal recognition particle interacting with the elongation-arrested ribosome. Nature 427: 808-814.

Han H, Schepartz A, Pellegrini M, Dervan PB. 1994. Mapping RNA regions in eukaryotic ribosomes that are accessible to methidiumpropyl-EDTA $\cdot \mathrm{Fe}(\mathrm{II})$ and EDTA $• \mathrm{Fe}(\mathrm{II})$. Biochemistry 33: 9831-9844.

Hashem Y, des Georges A, Dhote V, Langlois R, Liao HY, Grassucci RA, Hellen CU, Pestova TV, Frank J. 2013. Structure of the mammalian ribosomal $43 \mathrm{~S}$ preinitiation complex bound to the scanning factor DHX29. Cell 153: 1108-1119.

Hassouna N, Michot B, Bachellerie JP. 1984. The complete nucleotide sequence of mouse $28 \mathrm{~S}$ rRNA gene. Implications for the process of size increase of the large subunit rRNA in higher eukaryotes. Nucleic Acids Res 12: 3563-3583.

Henry Y, Wood H, Morrissey JP, Petfalski E, Kearsey S, Tollervey D. 1994. The $5^{\prime}$ end of yeast $5.8 \mathrm{~S}$ rRNA is generated by exonucleases from an upstream cleavage site. EMBO J 13: 2452-2463.

Horsey EW, Jakovljevic J, Miles TD, Harnpicharnchai P, Woolford JL Jr. 2004. Role of the yeast Rrp1 protein in the dynamics of pre-ribosome maturation. RNA 10: 813-827.

Jakovljevic J, Ohmayer U, Gamalinda M, Talkish J, Alexander L, Linnemann J, Milkereit P, Woolford JL Jr. 2012. Ribosomal proteins L7 and L8 function in concert with six $\mathrm{A}_{3}$ assembly factors to 
propagate assembly of domains I and II of $25 \mathrm{~S}$ rRNA in yeast $60 \mathrm{~S}$ ribosomal subunits. RNA 18: 1805-1822.

Jeeninga RE, Van Delft Y, de Graaff-Vincent M, Dirks-Mulder A, Venema J, Raue HA. 1997. Variable regions V13 and V3 of Saccharomyces cerevisiae contain structural features essential for normal biogenesis and stability of 5.8S and 25S rRNA. RNA 3: 476-488.

Jenner L, Melnikov S, Garreau de Loubresse N, Ben-Shem A, Iskakova M, Urzhumtsev A, Meskauskas A, Dinman J, Yusupova G, Yusupov M. 2012. Crystal structure of the 80 S yeast ribosome. Curr Opin Struct Biol 22: 759-767.

Kos M, Tollervey D. 2010. Yeast pre-rRNA processing and modification occur cotranscriptionally. Mol Cell 37: 809-820.

Lebaron S, Segerstolpe A, French SL, Dudnakova T, de Lima Alves F, Granneman S, Rappsilber J, Beyer AL, Wieslander L, Tollervey D. 2013. Rrp5 binding at multiple sites coordinates pre-rRNA processing and assembly. Mol Cell 52: 707-719.

Liang WQ, Fournier MJ. 1997. Synthesis of functional eukaryotic ribosomal RNAs in trans: development of a novel in vivo rDNA system for dissecting ribosome biogenesis. Proc Natl Acad Sci 94: 28642868.

Melnikov S, Ben-Shem A, Garreau de Loubresse N, Jenner L, Yusupova G, Yusupov M. 2012. One core, two shells: bacterial and eukaryotic ribosomes. Nat Struct Mol Biol 19: 560-567.

Michot B, Bachellerie JP. 1987. Comparisons of large subunit rRNAs reveal some eukaryote-specific elements of secondary structure. Biochimie 69: 11-23.

Michot B, Qu LH, Bachellerie JP. 1990. Evolution of large-subunit rRNA structure. The diversification of divergent D3 domain among major phylogenetic groups. Eur J Biochem 188: 219-229.

Musters W, Venema J, van der Linden G, van Heerikhuizen $H$, Klootwijk J, Planta RJ. 1989. A system for the analysis of yeast ribosomal DNA mutations. Mol Cell Biol 9: 551-559.

Musters W, Boon K, van der Sande CA, van Heerikhuizen H, Planta RJ. 1990. Functional analysis of transcribed spacers of yeast ribosomal DNA. EMBO J 9: 3989-3996.

Musters W, Concalves PM, Boon K, Raue HA, van Heerikhuizen H, Planta RJ. 1991. The conserved GTPase center and variable region V9 from Saccharomyces cerevisiae 26S rRNA can be replaced by their equivalents from other prokaryotes or eukaryotes without detectable loss of ribosomal function. Proc Natl Acad Sci 88: 1469-1473.

Nogi Y, Yano R, Dodd J, Carles C, Nomura M. 1993. Gene RRN4 in Saccharomyces cerevisiae encodes the A12.2 subunit of RNA polymerase I and is essential only at high temperatures. Mol Cell Biol 13: $114-122$.
Peculis BA, Greer CL. 1998. The structure of the ITS2-proximal stem is required for pre-rRNA processing in yeast. RNA 4: 1610-1622.

Rodriguez-Galan O, Garcia-Gomez JJ, Kressler D, de la Cruz J. 2015. Immature large ribosomal subunits containing the $7 \mathrm{~S}$ pre-rRNA can engage in translation in Saccharomyces cerevisiae. RNA Biol 12: 838-846.

Sahasranaman A, Dembowski J, Strahler J, Andrews P, Maddock J, Woolford JL Jr. 2011. Assembly of Saccharomyces cerevisiae 60S ribosomal subunits: role of factors required for $27 \mathrm{~S}$ pre-rRNA processing. EMBO J 30: 4020-4032.

Shajani Z, Sykes MT, Williamson JR. 2011. Assembly of bacterial ribosomes. Annu Rev Biochem 80: 501-526.

Spahn CM, Beckmann R, Eswar N, Penczek PA, Sali A, Blobel G, Frank J. 2001. Structure of the 80 S ribosome from Saccharomyces cerevisiae - tRNA-ribosome and subunit-subunit interactions. Cell 107: 373-386.

Sweeney R, Yao MC. 1989. Identifying functional regions of rRNA by insertion mutagenesis and complete gene replacement in Tetrahymena thermophila. EMBO J 8: 933-938.

Sweeney R, Chen L, Yao MC. 1994. An rRNA variable region has an evolutionarily conserved essential role despite sequence divergence. $\mathrm{Mol}$ Cell Biol 14: 4203-4215.

Talkish J, Campbell IW, Sahasranaman A, Jakovljevic J, Woolford JL Jr. 2014. Ribosome assembly factors Pwp1 and Nop12 are important for folding of $5.8 \mathrm{~S}$ rRNA during ribosome biogenesis in Saccharomyces cerevisiae. Mol Cell Biol 34: 1863-1877.

Taylor DJ, Devkota B, Huang AD, Topf M, Narayanan E, Sali A, Harvey SC, Frank J. 2009. Comprehensive molecular structure of the eukaryotic ribosome. Structure 17: 1591-1604.

Tutuncuoglu B, Jakovljevic J, Wu S, Gao N, Woolford JL Jr. 2016. The $\mathrm{N}$-terminal extension of yeast ribosomal protein L8 is involved in two major remodeling events during late nuclear stages of $60 \mathrm{~S}$ ribosomal subunit assembly. RNA doi: 10.1261/rna.055798.115.

Veldman GM, Klootwijk J, de Regt VC, Planta RJ, Branlant C, Krol A, Ebel JP. 1981. The primary and secondary structure of yeast $26 \mathrm{~S}$ rRNA. Nucleic Acids Res 9: 6935-6952.

Ware VC, Tague BW, Clark CG, Gourse RL, Brand RC, Gerbi SA. 1983. Sequence analysis of $28 \mathrm{~S}$ ribosomal DNA from the amphibian Xenopus laevis. Nucleic Acids Res 11: 7795-7817.

Waters AP, White W, McCutchan TF. 1995. The structure of the large subunit rRNA expressed in blood stages of Plasmodium falciparum. Mol Biochem Parasitol 72: 227-237.

Woodson SA. 2011. RNA folding pathways and the self-assembly of ribosomes. Acc Chem Res 44: 1312-1319. 

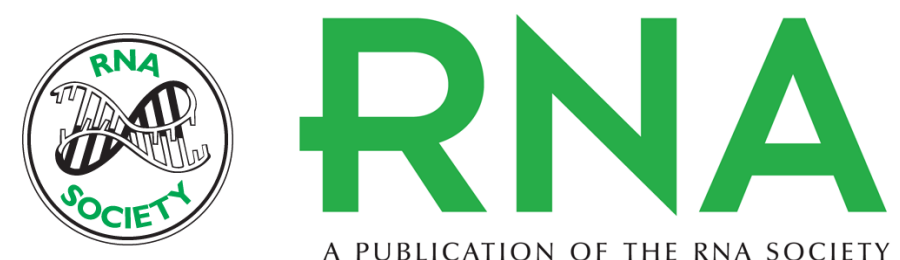

A PUBLICATION OF THE RNA SOCIETY

\section{Eukaryote-specific rRNA expansion segments function in ribosome biogenesis}

Madhumitha Ramesh and John L. Woolford, Jr.

RNA 2016 22: 1153-1162 originally published online June 17, 2016

Access the most recent version at doi:10.1261/rna.056705.116

\section{Supplemental http://rnajournal.cshlp.org/content/suppl/2016/06/17/rna.056705.116.DC1 Material}

References This article cites 51 articles, 19 of which can be accessed free at: http://rnajournal.cshlp.org/content/22/8/1153.full.html\#ref-list-1

Creative This article is distributed exclusively by the RNA Society for the first 12 months after the Commons License full-issue publication date (see http://rnajournal.cshlp.org/site/misc/terms.xhtml). After 12 months, it is available under a Creative Commons License (Attribution-NonCommercial 4.0 International), as described at http://creativecommons.org/licenses/by-nc/4.0/.

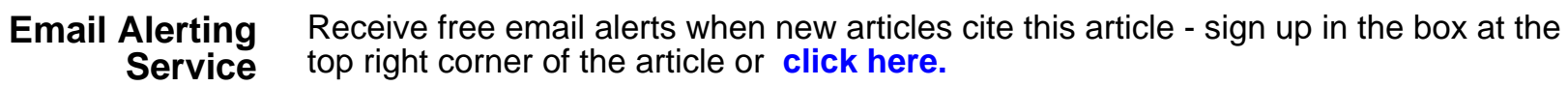

